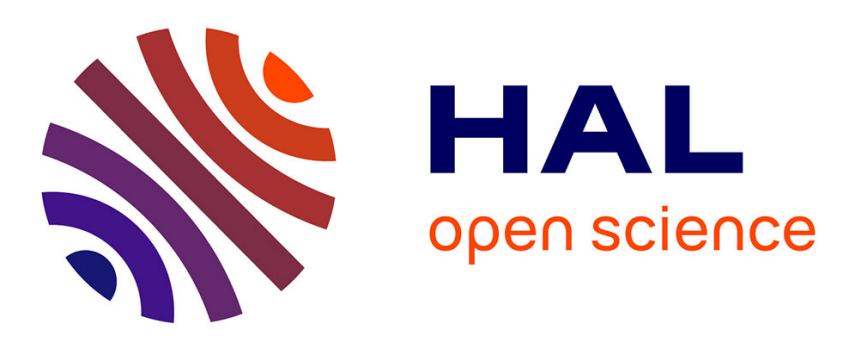

\title{
La pénétration des règles du procès pénal devant les juridictions de l'application des peines : état des lieux \\ Muriel Giacopelli
}

\section{To cite this version:}

Muriel Giacopelli. La pénétration des règles du procès pénal devant les juridictions de l'application des peines: état des lieux . Revue de Science Criminelle et de Droit Pénal Comparé, 2015, RSC 2015 4 pp.799-812. hal-01309574

\section{HAL Id: hal-01309574 https://hal.science/hal-01309574}

Submitted on 29 Apr 2016

HAL is a multi-disciplinary open access archive for the deposit and dissemination of scientific research documents, whether they are published or not. The documents may come from teaching and research institutions in France or abroad, or from public or private research centers.
L'archive ouverte pluridisciplinaire HAL, est destinée au dépôt et à la diffusion de documents scientifiques de niveau recherche, publiés ou non, émanant des établissements d'enseignement et de recherche français ou étrangers, des laboratoires publics ou privés. 


\section{LA PENETRATION DES RÈGLES DU PROCÈS PÉNAL DEVANT LES JURIDICTIONS DE L'APPLICATION DES PEINES : ETAT DES LIEUX}

Muriel GLACOPELLI, Professeur à Aix-Marseille Université, LDPSC (EA 4690)

Directrice de l'ISPEC

\section{INTRODUCTION.}

1- La notion de procès, couramment usitée et renvoyant étymologiquement au terme latin «processus », demeure encore à maints égards une notion difficile à cerner. C'est ainsi que le Grand Robert de la langue française définit le procès comme «le litige soumis par les parties à une juridiction». Quant au Vocabulaire juridique Cornu, il retient sensiblement la même acception du procès soit le «litige soumis à un tribunal; contestation pendante devant une juridiction». Pour le Littré, le procès est «l'instance devant un juge, sur un différend entre deux ou plusieurs parties». L'on peut dégager deux grands critères définitoires du procès : l'un renvoie à la matière du procès ou l'objet du litige, l'autre à la manière dont la juridiction tranche le litige. Si le Code de procédure civile consacre de nombreux articles à la détermination du litige ${ }^{1}$, le Code de procédure pénale se montre silencieux préférant renvoyer à l'action. Deux actions y sont visées : l'action publique (art. $1^{\text {en }}$ ) qui est définie comme l'action pour l'application des peines et l'action civile pour la réparation du préjudice né de l'infraction (art. 2 Cpp). Or, aucune de ces deux actions ne correspond véritablement à l'action menée devant les juridictions de l'application des peines ${ }^{2}$. Ce qui a pu faire dire qu'il s'agissait pour cette dernière d'une action «à fin pénale ${ }^{3}$ » dont l'objet reste encore à déterminer. Devant ces constatations liées à la faiblesse définitoire du Code de procédure pénale dans le domaine post-sentenciel, le procès sera envisagé

\footnotetext{
${ }^{1}$ Les articles 4 et $5 \mathrm{Cpc}$ forment à eux seuls une section intitulée « l'objet du litige ».

${ }^{2}$ En ce sens, E. BONIS-GARÇON ET V. PELTIER, Droit de la peine, Dalloz $2^{\mathrm{ème} e}$ éd,

${ }^{3} \mathrm{~V}$. ibidem, no 925 par comparaison avec l'action à fin publique. Sur cette notion V. P. CONTE, la nature juridique des procédures alternatives aux poursuites: de l'action publique à l'action à fin publique, in Mélanges offerts à R. Gassin, PUAM, 2007, p. 189.
} 
comme la manière d'organiser «le processus pénal » devant les juridictions de l'application des peines.

2- Il est alors logique de dater la naissance du droit de l'application des peines à la consécration des juridictions de l'application des peines par la loi du 9 mars 2004 portant adaptation de la justice aux nouvelles formes de criminalité. Certes la loi du 9 mars 2004 n'est que l'aboutissement d'une évolution initiée par la loi du 15 juin 2000 ayant juridictionnalisé l'exécution des peines, qui est selon le doyen Cornu, "le procédé consistant à attribuer à des actes qui ne le comporteraient pas normalement la qualification d'acte juridictionnel afin de leur en étendre le régime de ce dernier $»^{5}$. Il n'est pas nécessaire de revenir sur un phénomène que la doctrine a largement étudié ${ }^{6}$ et dont les conséquences juridiques ont été l'ouverture des voies de recours. Très rapidement des opinions dissidentes se sont fait entendre montrant les incohérences de la juridictionnalisation de l'exécution des sanctions pénales ${ }^{7}$, comme étant le signe des aléas d'une juridictionnalisation lente et progressive sans théorisation préalable.

3-Si la loi du 9 mars 2004 a pour l'essentiel fait œuvre de rationalisation des procédures de l'application des peines, le législateur n'a eu de cesse d'empiler les textes créant notamment de nouvelles catégories de procédures. Il en est ainsi des procédures simplifiées en aménagement de peines issues de la loi du 24 novembre 2009, dite loi pénitentiaire, qui ont développé pour les condamnés en fin de peines des procédures applicables par l'administration pénitentiaire (Cpp art. 723-20) sous le contrôle du procureur de la République. Sous couvert d'exigences liées à la gestion du flux carcéral, ces procédures déjudiciarisées ont témoigné d'une certaine méfiance à l'encontre du juge. Les réformes précédant la promulgation de la loi du 15 août 2014 relative à l'individualisation

\footnotetext{
${ }^{4}$ S. Guinchard ET J. Buisson, Procédure pénale, Lexis Nexis, $10^{\mathrm{ème}}$ éd. $\mathrm{n}^{\circ} 2$.

${ }^{5}$ CORNU, Vocabulaire juridique, Association H. Capitant, éd. PUF, vo Juridictionnalisation.

${ }^{6}$ P. PONCELA, «Le chantier de la juridictionnalisation est ouvert », Rev. sc. crim. 2000, 887 ; P. FAUCHER, «La juridictionnalisation de l'application des peines : une révolution tranquille », Rev. pénit. 2001, pp215-223 ; M. Herzog-Evans, "Juridictionnalisation de l'application des peines : le bilan », Rev. pénit. 2007, pp. 175-182; E. BONIS-GARCON, «la juridictionnalisation de l'application des peines », Dr Pénal 2007, étude p. 28-31.

${ }^{7}$ E. BONIS-GARCON, Les incohérences de la juridictionnalisation au stade de l'exécution des sanctions pénales, in La réforme du Code pénal et du Code de procédure pénale- Opinio doctorum, Dalloz, Coll « Thèmes et commentaires », 2009 p. 313 et ss.
} 
des peines et l'efficacité de la sanction pénale ${ }^{8}$, ont soumis la matière à des injonctions en sens contraire. En outre, le législateur a rapidement repris l'habitude de créer une procédure par mesure nouvelle d'aménagement des peines comme ce fut le cas de la création de la Surveillance judiciaire des personnes dangereuses ${ }^{9}$ ou du placement sous surveillance électronique. Pire encore, l'introduction de la rétention et la surveillance de sûreté dans le Code de procédure pénale ${ }^{10}$ s'est accompagnée de la création de nouvelles juridictions ad'boc rendant plus complexe les critères de répartition des compétences d'attribution. Pour ces diverses raisons, les règles applicables devant les juridictions de l'application des peines sont en grande partie dérogatoires aux règles du procès pénal. C'est ainsi que devant la CHAP la comparution du condamné n'est pas prévue (Cpp art 712-13 et D. 49-42) ou bien encore la règle de la publicité des débats est le plus souvent écartée. Il est vrai que par l'ensemble de ces réformes, le législateur n'a guère cherché à transposer à la phase de l'application des peines les garanties accordées tout au long du procès pénal ${ }^{11}$, mais s'est davantage inquiété du sort de la personne condamnée en lui octroyant des droits nouveaux.

4- Si les avancées ont été considérables, le maintien de règles procédurales spécifiques ne trouvent plus guère de justifications, notamment sous la pression du droit européen. Il est alors de plus en plus contestable d'écarter les grands principes directeurs du procès pénal devant les juridictions de l'application des peines. Parmi eux se trouvent notamment les règles du procès équitable au sens de l'article 6 Conv. EDH. Son application devant les juridictions de l'application des peines est encore controversée en raison du champ de l'article 6, lequel ne porte que sur « le bien fondé de toute accusation en matière pénale». Il apparaît toutefois que certaines décisions prises par les juridictions de l'application des peines, surtout lorsqu'il s'agit de statuer sur la révocation d'une mesure d'aménagement de peine, seraient susceptibles de déclencher l'application de l'article $6^{12}$.

\footnotetext{
${ }^{8}$ La loi du 15 août 2014 a en effet abrogé les procédures simplifiées des condamnés en fin de peine pour les remplacer par la nouvelle procédure de libération sous contrainte. ${ }^{9}$ L. n 2005-1549, 12 déc. 2005,

${ }^{10}$ L. GREGOIRE, «Les mesures de sûreté : essai sur l'autonomie d'une notion », Thèse Aix-enProvence, dec. 2014, dir. Ph. Bonfils.

${ }^{11}$ En ce sens : P. FAUCHER, Juris classeur, Fasc. 40 : les juridictions de l'application des peines, mise à jour 12 fév. 2015.

${ }^{12}$ En ce sens : P. FAUCHER, Jusrisclasseur Procédure Pénale, Fasc. 20 : Juridictions de l'application des peines, Composition,, compétences, nº 18, mise à jour du 5 mai 2014.
} 
5- Aussi assiste-t-on depuis quelques années à de timides avancées devant les juridictions de l'application des peines par emprunt aux règles du procès pénal tant dans le droit au procès en application des peines (I) que dans le doit du procès en application des peines (II).

\section{I/ LES REGLES DU PROCES PENAL APPLICABLES AU DROIT AU PROCES DEVANT LES JURIDICTIONS DE L'APPLICATION DES PEINES}

6- Le droit au procès s'entend du droit au juge qui mobilise le droit conventionnel et renvoie à la notion de procès équitable dans ses diverses dimensions. Malgré les réserves sur l'applicabilité de l'article $6 \$ 1$ de la Conv.EDH déjà signalées, les exigences de l'article 6, bien qu'imparfaitement transposées, ne sont pas totalement étrangères au procès devant les juridictions de l'application des peines. C'est ainsi que l'on peut affirmer que sous l'effet de la juridictionnalisation, le droit au juge semble pour l'essentiel garanti (A), tandis que l'on assiste à la pénétration des garanties procédurales devant les juridictions de l'application des peines (B).

\section{$\underline{\text { A/ le droit au juge dans l'application des peines. }}$}

7- Le droit au juge, c'est d'abord le droit à un premier juge de pleine juridiction. C'est ensuite le droit à un deuxième juge, celui de l'appel. La généralisation des voies de recours, comme conséquence de la juridictionnalisation, a conduit le législateur du 9 mars 2004 à répondre aux exigences du droit à un tribunal au sens conventionnel, par la fondation des juridictions de l'application des peines de $1^{\text {er }}$ et de second degré. C'est qu'en effet, si le JAP a été crée en 1958, il n'était envisagé guère plus que comme un passeur. Servant de relais au pouvoir exécutif dans l'exécution de la sentence pénale, les décisions du JAP avaient la nature de décisions d'administration judiciaire. Supposées ne pas faire grief aux personnes détenues, elles n'étaient pas soumises à recours. La juridictionnalisation de l'exécution des peines a eu pour effet d'ouvrir les voies de recours, d'abord devant les juridictions existantes, soit devant la chambre des appels correctionnels ${ }^{13}$, puis devant la chambre de l'application des peines créées à cet effet. A cet égard la garantie du droit au juge, en ce qu'il est relayé par le

13 J. COSTE et P. REMILLIEUX, Exécution des peines : faute de grives... petits décrets, Répertoire de droit pénal et procédure pénal, Dalloz, mai 2002, p. 3. 
principe de «second degré de juridiction $»^{14}$, a été une condition essentielle à l'émergence du droit de l'application des peines.

8-Cependant, le droit au juge a une dimension plus large que la seule existence de voies de recours, lesquelles en soi doivent être envisagées comme une exigence fondamentale minimale dans toute société démocratique. L'affirmation s'entend aussi comme d'un égal accès au juge. A cet égard, la procédure devant les juridictions de l'application des peines n'obéit pas aux règles traditionnelles du procès équitable. L'architecture de l'application des peines présente une singularité en ce qu'elle est composée, au premier degré, du JAP et du Tribunal d'Application des Peines (TAP). Le TAP crée par la loi du 9 mars 2004, a été instauré aux côtés du JAP, pour le traitement des mesures d'individualisation des condamnés aux longues peines. Sa genèse explique pour partie la compétence d'attribution qui est la sienne, qui est strictement déterminée par les textes et qui est exclusive pour certaines mesures comme le relèvement de la période de sûreté ${ }^{15}$, les réductions de peines exceptionnelles ${ }^{16}$ ou encore le relèvement du SSJ à l'issue d'une période de trente ans ${ }^{17}$. Eu égard leur composition respective, juge unique pour l'un et collégialité pour l'autre, l'on aurait pu s'attendre conformément aux règles procédurales traditionnelles que le premier rende des ordonnances et le second des jugements. Or, le JAP et le TAP partagent la compétence juridictionnelle en la forme de jugements, alors que seul le JAP rend des ordonnances. Mais selon que la décision est rendue sous forme d'ordonnance (JAP) ou sous forme de jugement (JAP et TAP), les garanties procédurales et les voies de recours opèrent selon des règles différentes.

9-C'est ainsi que la non-conformité de l'article D. 49-39 du Code de procédure pénale à l'article 6 de la Conv.EDH, en ce qu'il réserve au ministère public des délais supplémentaires en cas d'appel incident contre des ordonnances ${ }^{18}$ ou des jugements ${ }^{19}$ rendus par le JAP, a été soulevée devant la juridiction administrative. La procédure portait en l'espèce sur la contestation de la réduction du

${ }^{14}$ Le principe de second degré de juridiction ne peut être écarté, que lorsqu'un texte le prévoit expressément. C'est ainsi que le Cpp n'a guère connu que de l'exception de l'appel des arrêts de Cour d'assises jusqu'à la promulgation de la loi du 15 juin 2000.

${ }^{15}$ Cpp art. $720-4$

${ }^{16}$ Cpp. art. $721-3$

${ }^{17}$ Cpp art. 131-36-1

${ }^{18}$ Un délai de $24 \mathrm{~h} 00$ supplémentaire est réservé au ministère public pour former appel incident en cas d'appel du détenu.

${ }^{19}$ Un délai de 5 jours supplémentaires est accordé au Ministère public dans les mêmes conditions. 
crédit de peine pour mauvaise conduite prononcée par voie d'ordonnance par le JAP. La procédure des CRP est pour une large part dérogatoire au droit commun de la procédure pénale. Le législateur a en effet entendu préserver un certain équilibre entre le respect des principes attachés à la juridictionnalisation de l'exécution des peines et la fluidité du traitement d'un contentieux de masse, en réservant au CRP une procédure écrite, sans débat contradictoire oral au cours d'une audience publique. Ce dispositif n'a pas tardé à susciter des contestations sur sa conformité aux normes supérieures. C'est ainsi que pour répondre à la requête du condamné, le Conseil d'Etat s'est au préalable penché sur la qualification pénale au sens conventionnel de la procédure de l'article 721 du Cpp.

10-Par sa portée, l'arrêt rendu par le Conseil d'Etat le 24 octobre $2014^{20}$ déborde largement la question initiale de procédure pour (ré)ouvrir le débat sur la question de la nature des réductions de peine et subséquemment le rapport de la faute disciplinaire à la faute pénale. Le retrait des réductions de peine a deux origines possibles que sont d'une part une sanction disciplinaire en prison qui motivera la saisine du JAP et d'autre part, la commission d'une nouvelle infraction. Le Conseil d'Etat ayant récemment jugé que le retrait de CRP faisant suite à une nouvelle condamnation de l'intéressé n'est pas contraire au principe ne bis in idem ${ }^{21}$, les effets de la faute disciplinaire sur les réductions de peine en la forme de retrait de peine posent en revanche la question de l'applicabilité de l'article $6^{22}$. Le Conseil d'Etat a jugé « qu'eu égard à leurs conséquences pour la durée de l'emprisonnement du condamné, les décisions de retrait de la réduction de peine sur laquelle le détenu était en droit de compter en application de l'article 721 du code de procédure pénale, que prend le juge de l'application des peines sur le fondement de ces dispositions, doivent être regardées comme relevant de la matière pénale, au sens des stipulations de l'article 6 de la convention EDH dont le requérant peut, dès lors, utilement se prévaloir à l'encontre des dispositions litigieuses. En l'espèce, en réservant au ministère public la faculté de former appel incident, les dispositions contestées méconnaissent le principe de l'égalité des armes garanti par ces stipulations ${ }^{23}$. En agrégeant les CRP à la matière pénale, le Conseil d'Etat a pris le contre-pied de la décision QPC rendue par le Conseil Constitutionnel le 11

${ }^{20}$ CE 24 oct. 2014 req. n 368580, AJpénal 2014, n³8, p. 2176, note J-P Céré ; Gaz. Pal. 18 déc. 2014 n $^{\circ} 352$, p. 20, obs. Roussel.

${ }^{21}$ CE 25 mars 2015, req. n 374401.

${ }^{22} \mathrm{~V}$. déjà sur la question : J-P CÉRÉ, l'article 6 de la convention européenne des droits de l'homme et le procès disciplinaire en prison, JCP G 2001, I. 316.

${ }^{23}$ CE 24 oct. 2014 op. cit. 
juillet 2014 ${ }^{24}$. Le Conseil Constitutionnel a affirmé que le retrait d'un CRP en cas de mauvaise conduite du condamné, qui a pour conséquence que le condamné exécute totalement ou partiellement la peine telle qu'elle a été prononcée par la juridiction de jugement ne constitue ni une peine, ni une sanction ayant le caractère de punition.

11-Ces décisions sont à rapprocher d'un arrêt rendu par la Chambre criminelle de la Cour de cassation le 15 avril $2015^{25}$. Pour confirmer la décision du JAP, retirant au condamné 25 jours de CRP, une chambre d'application des peines a énoncé notamment, qu'en insultant le personnel pénitentiaire et en détenant des objets prohibés, M.H n’a pas eu la bonne conduite exigée par la loi. «En l'état de ces seules énonciations, qui suffisent à caractériser la mawvaise conduite du condamné en détention, au sens de l'article 721 alinéa 3 CPp, le Président de la CHAP a justifié sa décision de supprimer un avantage que le condamné ne pouvait espérer conserver malgré son mawvais comportement, et qui entraîne, pour celui-ci, aucune privation de liberté distincte de la peine en cours d'exécution, sans méconnaître les articles 6 et 13 de la Conv. EDH. En effet, d'une part, le CRP est inscrit à l'écrou, en début d'exécution de cette peine, à titre précaire, sous condition pour le condamné, qui en est informé, d'observer la bonne conduite nécessaire au fonctionnement normal de l'établissement carcéral, d'autre part, le retrait total ou partiel de ce crédit est décidé par un juge, qui n'est pas lié par la décision disciplinaire prise par l'administration pénitentiaire, et dont l'ordonnance est susceptible d'appel, la décision du Pt de la CHAP pouvant ensuite faire l'objet d'un pourvoi en cassation, de sorte que sont pleinement assurés l'exercice des droits de la défense et l'équité de la procédure $»^{26}$.

12-L'arrêt rendu par la chambre criminelle appelle deux observations liminaires. La première concerne le soin avec lequel la chambre criminelle a rédigé son argumentaire, rompant avec l'habitude prise jusqu'alors par la haute juridiction d'écarter in abstracto l'application de l'article 6 à la procédure observée devant les juridictions de l'application des peines ${ }^{27}$. L'examen concret des dispositions de droit interne au regard des exigences du procès équitable, même pour conclure à leur conformité, dénote déjà d'une évolution de la chambre criminelle sur sa propre jurisprudence. La

\footnotetext{
${ }^{24}$ Cons. const. 11 juill. 2014, n 2014-408 QPC, D. 2014, 1500 ; AJ pénal 2014 ;545, obs. M. HerzogEvans ; Dr Pénal oct. 2014, p. 35, note E. Bonis-Garçon.

25 crim. 15 avr. 2015, n¹4-80.417 ; D. 2015. 925 ; D. 2015, 1401, obs. B. Laurent ; AJ Pénal 2015, comm. 104, E. Bonis-Garçon.

${ }^{26}$ ibidem.

${ }^{27}$ V. par exemple devant le Président de la CHAP : Cass. crim. 31 oct. 2006, AJPénal 2007, 38, obs. M. Herzog-Evans
} 
seconde concerne le visa des articles 6 et celui inédit de l'article 13 de la Conv. EDH. Quant à l'analyse, elle est le reflet inversé de celle proposée par le conseil d'Etat en raison d'une appréciation sémantique différente des deux ordres juridictionnels dans la définition de la peine, conventionnelle pour l'un et constitutionnelle pour l'autre. Le Conseil d'Etat s'attache aux effets des CRP, soit le calcul de réduction de peine que la personne détenue serait en droit d'attendre comme nourrissant une espérance légitime de recouvrer sa liberté et comme telle, susceptible d'avoir une incidence sur la portée de la peine. Mais c'est oublier comme le rappelle la Cour de cassation, que ce droit est sous condition de l'absence de mauvais comportement de la personne détenue. Autrement dit, la soumission du dispositif à une logique comptable par la réforme de $2004^{28}$, substituant aux réductions de peines ordinaires les CRP, n'a fait que mettre en adéquation le droit à la pratique, sans pour autant changer la nature des réductions de peines. Pas plus qu'avant la personne détenue n'a de droit acquis aux CRP.

13-Cependant et quelques mois après sa décision du 24 octobre 2014, le Conseil d'Etat a rejeté le 18 février $2015^{29}$ la requête en annulation tendant à l'abrogation des articles D. 115-7 à D. 115-12 pour violation des blocs de constitutionnalité et de conventionnalité et méconnaissance du principe de légalité en ce que la notion de «mauvaise conduite» susceptible de fonder le retrait de CRP insuffisamment claire et précise serait soumise à une interprétation fluctuante. Faisant machine arrière par rapport au processus enclenché d'intégration aux règles du procès équitable, le Conseil d'Etat rappelle que le processus de juridictionnalisation trouve sa limite dans la nature disciplinaire de la décision prise par le chef d'établissement, laquelle ne s'impose pas au JAP lorsqu'il fixe le quantum du retrait de CRP. Par voie de conséquence, il appartient au pouvoir réglementaire de préciser la notion de «mauvaise conduite », laquelle est suffisamment définie dans le règlement intérieur type, auquel la loi pénitentiaire du 24 novembre 2009 renvoie pour la détermination des droits et devoirs des personnes détenues. Malgré les divergences d'analyse entre le Conseil d'Etat et la Cour de Cassation, et subséquemment la difficulté à saisir dans toutes ses dimensions la problématique de l'application de l'article 6 devant les juridictions de l'application des peines, le fait même que l'article 6 s'invite au débat en droit de la peine est annonciateur d'une évolution prochaine confortée par d'autres décision renforçant les garanties procédurales devant les juridictions de l'application des peines.

\footnotetext{
${ }^{28}$ P. PONCELA, « Peine et crédit. Loi du 9 mars 2004 », rev. sc. crim. 2004 nº 455.

${ }^{29}$ CE 18 février 2015, n³75765, AJ Pénal avril 2015, 195, note M. Herzog-Evans et E. Péchillon.
} 


\section{$\underline{B}$ / La montée des garanties procédurales}

14- Le procès pénal offre conformément aux standards internationaux une procédure publique et équitable, cette dernière garantie renvoyant à la fois à une notion autonome et des applications particulières comme le respect des droits de la défense ${ }^{30}$. C'est au demeurant la formule « de débat contradictoire » que le législateur a préférée à celle d'audience pour la procédure dite de «droit commun » de l'article 712-6 et 712-7, en raison justement de l'écart existant avec les règles du procès pénal. L'on ne saurait trop insister sur le caractère dérogatoire au droit commun de la procédure devant les juridictions de l'application des peines. C'est ainsi que la publicité des débats est largement écartée, tandis qu'en appel la comparution du condamné n’est de droit que lorsque la décision de premier ressort a été prise en l’absence du condamné dûment convoqué (Cpp art. 712-9 al.2).

15- Le principe de la comparution du condamné est devenu un enjeu majeur du débat sur la normalisation des règles du procès devant les juridictions de l'application des peines. C'est ainsi que sauf lorsqu'elle est de droit en l'absence de comparution du condamné en première instance, la comparution devant la CHAP est laissée à la discrétion de la juridiction d'appel. La chambre criminelle de la Cour de cassation a rendu le 15 avril $2015^{31}$ un arrêt à la solution inédite qui rompt avec sa jurisprudence sur la comparution facultative du condamné devant la CHAP. Elle a affirmé que le respect des principes du contradictoire et de l'équilibre des droits des parties interdit à la chambre de l'application des peines se prononçant sur une demande de révocation de libération conditionnelle de statuer sans que le condamné qui en fait la demande ait été mis en mesure de comparaître à l'audience. En effet, la Cour de cassation censure au motif qu'au cours de la procédure en appel, le requérant n'avait pas été averti de son droit à demander sa comparution devant la juridiction d'appel pour se défendre. La chambre criminelle semble prendre en considération certaines critiques déjà formulées par la doctrine relativement, dans un contexte socio-économique fragile, à l'évolution des données du litige et de la situation du requérant devant la cour d'appel ${ }^{32}$.

16-L'arrêt a été rendu sous la forme d'un arrêt de principe, au triple visa des articles 6 de la Conv.

\footnotetext{
${ }^{30}$ En ce sens. J. BuISSON et S. GiNCHARD, Procédure pénale, op. cit. n 435 et ss.

${ }^{31}$ Cass. crim. 15 avr. 2015, n¹4-82622, Dr Pénal 2015, nº 6, comm. 91, obs. E. Bonis-Garçon.

${ }^{32}$ En ce sens : E. SENNA, « de l'individualisation de la peine au second degré de juridiction postsentenciel », RPDP 2015, p. 37.
} 
EDH, préliminaire et 733 Cpp. La chambre criminelle dit applicable l'article 6 devant les juridictions de l'application des peines, alors qu'elle avait précédemment justifié le refus de transmission d'une QPC portant sur la conformité de l'article 712-13 Cpp aux articles 9 et 16 de la DDHC au motif que le rejet d'une demande de libération conditionnelle n'est pas une sanction pénale au sens constitutionnel $^{33}$. Dans un autre arrêt rendu, le 20 mars 2013, la chambre criminelle a apprécié le niveau de garantie des droits fondamentaux sous le seul angle processuel du double degré de juridiction ${ }^{34}$. La cour de cassation a ainsi considéré que le niveau des droits de la défense peut être allégé en appel, dès lors que la comparution de l’intéressé est de droit devant le premier juge et qu'il appartient à la CHAP statuant après débat contradictoire au cours duquel l'avocat du condamné est entendu dans ses observations, d'entendre le condamné, le cas échéant à sa demande par visioconférence ou par un de ses membres dans l'établissement pénitentiaire où il se trouve.

16-A l'aune d'une jurisprudence non stabilisée, il est difficile de déterminer la portée exacte de l'arrêt rendu le 15 avril dernier. Cet arrêt ouvre néanmoins certaines perspectives et l'on se risquera à creuser quelques pistes. Jusqu'alors la Cour de cassation contrôlait l'effectivité de la comparution de la personne condamnée uniquement dans les hypothèses où celle-ci est de droit. C'est ainsi que dans cette hypothèse, la Cour de cassation a considéré que l'obligation de comparution n'avait pas été respectée par la CHAP qui a ignoré le changement d'adresse signalé par le condamné en ne mettant pas le condamné en mesure de comparaître devant elle ${ }^{35}$. Peut-on considérer que la solution nouvellement adoptée crée au profit du condamné un droit à la comparution personnelle devant la Cour d'appel quelle que soit les hypothèses de droit ou facultative envisagées. Ou bien la solution est-elle limitée au seul contentieux de la libération conditionnelle ? Quant à son fondement, peut-on aller jusqu'à admettre que s'agissant de la révocation ou du retrait d'une mesure, la question à trancher pourrait être assimilée «à l'examen du bien-fondé d'une accusation en matière pénale » ? La $\mathrm{CEDH}$ l'a déjà admis pour certaines questions spécifiques liées à l'exécution d'une décision ${ }^{36}$. Cette solution doit donc emporter l'adhésion. Ce d'autant plus, qu'il est de moins en moins légitime de

\footnotetext{
${ }^{33}$ Cass. crim. 19 janv. 2011, 10-85.354, QPC non-lieu à renvoi.

${ }^{34}$ Cass. crim. 20 mars 2013, n 13-90001 QPC, AJ pénal 2013, p. 486, note M. Herzog-Evans.

35 Cass. crim. 18 décembre 2013, n¹3-80918, AJ pénal 2014, Jp 197, obs. M. Herzog-Evans, Dr pénal 2014, chron. « un an de droit de la peine », n 35, Obs. E B-G

${ }^{36}$ L'article 6 pourrait en effet sous certaines conditions s'appliquer devant les juridictions des peines comme l'a déjà admis la CEDH pour des questions spécifiques relatives à l'exécution de certaines décisions : CEDH, 19 mars 1997, Hornsby c/ Grèce JCP G 1997, II, 22949 ; D. 1998,p. 74.
} 
limiter la comparution de la personne devant la CHAP en raison des derniers obstacles d'ordre pratique qui ont été levés sous l'effet de la généralisation du recours à la visioconférence préconisé par le contrôleur général des lieux de privation de liberté ${ }^{37}$.

16- Par ailleurs, la chambre criminelle pour corriger en quelque sorte les effets délétères des procédures dites hors débat, veille au strict respect des droits de la défense lorsque le débat contradictoire prévu par les textes n'a pas été écarté d'un commun accord des parties ${ }^{38}$, qu'il s'agisse de la régularité de la convocation de l'avocat au débat contradictoire ${ }^{39}$, ou de l'affirmation inédite que l'avocat a la parole en dernier ${ }^{40}$. De même, la Cour de Cassation exerce un contrôle rigoureux sur le respect des délais dans lesquels le condamné peut présenter ses observations lors de l'appel. Sauf hypothèse de l'urgence ${ }^{41}$, l'article 712-2 Cpp prévoit que le Pt de la CHAP statue sur l'appel des ordonnances du JAP, par ordonnance motivée rendue au vu des observations écrites du parquet et du condamné, qui bénéficie d'un mois à compter de la date d'appel pour communiquer ses observations. Non seulement la chambre criminelle interdit à la juridiction d'appel de se prononcer avant cette date ${ }^{42}$, mais exige du juge qu'il fasse également état dans sa décision des observations transmises par le condamné afin de mettre la cour de cassation en mesure d'exercer son contrôle ${ }^{43}$.

17-La modélisation du procès devant les juridictions de l'application des peines invite enfin à la généralisation du principe de publicité dont on sait qu'il est inversé devant elles. Les prémices d'un changement ont été annoncées par le législateur alors que la jurisprudence semble peu encline à une telle évolution. C'est ainsi que la chambre criminelle a refusé de faire droit à la requête d'une personne condamnée faisant valoir la faiblesse du fondement juridique permettant d'asseoir l'éviction de la publicité en appel. Si en effet une telle exclusion est fondée sur des dispositions

\footnotetext{
${ }^{37}$ Avis du contrôleur général des lieux de privation de liberté du 14 octobre 2011 relatif à l'emploi de la visioconférence à l'égard des personnes privées de liberté, JO 9 nov. 2011.

${ }^{38}$ La procédure hors débat ne peut que concerner l'octroi de la mesure et non son retrait qui nécessite un minimum de garanties.

${ }^{39}$ Cass. crim. 26 juin 2013, no 12-83.528, AJ pénal 2014 p. 46,

${ }^{40}$ Cass. crim. 10 déc. 2014 n ${ }^{\circ}$ 14-81056, AJ Pénal 2015 p.330.

${ }^{41}$ V. pour une illustration récente : Cass. crim. 15 mai 2013, no 12-85.586.

${ }^{42}$ Cass. crim. 6 sept. 2006, n 06-82205 ; Bull ; crim. 2006 n 211 ; Cass. crim. 18 juin 2008, n 07-82076, BC $2008 n^{\circ} 158$.

${ }^{43}$ Cass. crim. 26 juin 2013, n 13-80194, Dr pénal 2014, chronique du droit de la peine, $\mathrm{n}^{\circ} 34$, obs. E. BonisGarçon.
} 
expresses de nature législative, qu'il s'agisse de la décision prise en chambre du Conseil ${ }^{44}$ ou des ordonnances du JAP en CAP, aucune disposition légale ne permet de fonder en appel une telle exception contraire au principe général processuel consacré tant par l'article 592 Cpp, que l'article 6 de la Conv.EDH. En effet, les règles touchant à la publicité des débats sont des règles de procédure pénale, qui sont évoquées en appel par le seul article D. 49-42 Cpp en violation de l'article 34 de la constitution et de la répartition des blocs de compétence. La chambre criminelle y répond en rattachant de manière artificielle l'exception à l'article 711 Cpp. Ce qui ne trompe guère en ce que cet article, non spécifique à la matière, a trait aux différents incidents contentieux ${ }^{45}$ !

18-Paradoxalement, en mettant en lumière le rôle croissant des juridictions de l'application des peines et celui du JAP artisan de la mise en œuvre de la nouvelle peine de contrainte pénale, la réforme du 15 août 2014 relative à l'individualisation de la sanction pénale et l'efficacité des peines ${ }^{46}$ invite à une réflexion sur la recomposition des institutions pénales et l'application des règles processuelles devant les juridictions de l'application des peines. C'est ainsi que la procédure décrite pour la mise en œuvre de la contrainte pénale par le JAP ne coïncide pas parfaitement avec les procédures devant les juridictions de l'application des peines et se rapproche davantage des règles du procès pénal. L'article 713-43 al. 2 Cpp pour la fixation initiale des obligations de la contrainte pénale, s’il ne prévoit toujours pas de débat contradictoire suppose néanmoins une comparution devant le JAP. Quant à la révocation de la mesure comme sanction du non respect des obligations mises à la charge du condamné au titre de la contrainte pénale, le Pt du tribunal correctionnel saisi par le JAP, statue publiquement conformément aux dispositions de l'article 712-6 du Code de procédure pénale (Sic)! Or, si l'article 712-6 Cpp prévoit bien un débat contradictoire devant le CHAP, celui-ci se fait en chambre du conseil. La conformité visée par le législateur du 15 juin est-elle une simple erreur de plume ou augure-t-elle d'une mise en conformité avec les règles du procès pénal applicables au droit du procès devant les juridictions de l'application des peines?

\footnotetext{
${ }^{44}$ Cpp art. $712-6$ et $712-7$

45 Cass.crim. 15 avr. 2015, op. cit. ; Cass.crim. 14 oct. 2014, n 13-85.365, AJ Pénal 2015, p. 267, obs. M. Herzog-Evans.

${ }^{46}$ G. BeAusSONIE, chron. législ. RSC 2014, 809; M. GIACOPELli, La loi du 15 août 2014 relative à l'individualisation des peines et renforçant l'efficacité des sanctions pénales : un rendez-vous manqué, in Dossier la loi du 15 août 2014, AJ pénal 2014, 456 ; V. PELTIER, les »boîtes à outils » de Madame Taubira, JCP G 2014, aperçu rapide $n^{\circ}$ 883, p. 1510-1513, J. PRADEL, Un législateur bien imprudent, A propos de la loi $n^{\circ}$ 2014-896 du 15 août 2014, JCP 2014, I, 1642 ; J-H ROBERT, Punir dehors. Commentaire de la loi n 2014-896 , Dr pénal 2014, étude 16.
} 


\section{II/ LES RÈGLES DU PROCÈS PENAL APPLICABLES AU DROIT DU PROCÈS DEVANT LES JURIDICTIONS DE L'APPLICATION DES PEINES}

19- Le droit du procès renvoie aux règles fixes qui en organisent son déroulement ${ }^{47}$. Ce sont les éléments fédérateurs de l'instance devant les juridictions pénales. Deux éléments objectifs du procès pénal retiendront notre attention dans cette étude. Il s'agit d'une part de l'office du juge (A) et de sa localisation (B).

\section{A/ l'office du juge}

20- Conformément aux dispositions de l'article 712-1 cpp, le JAP et le TAP constituent les juridictions de l'application des peines de premier degré qui sont chargées dans les conditions légales, de fixer les principales modalités de l'exécution des peines privatives de liberté ou de certaines peines restrictives de liberté, en orientant et en contrôlant les conditions de leur application. A cette fin, le Code de procédure pénale reconnaît de larges pouvoirs aux juridictions de l'application des peines pour l'accomplissement de leur mission. C'est ainsi que pour l'instruction du dossier devant elles, les juridictions de l'application des peines disposent de très larges pouvoirs d'investigation tant géographiques ${ }^{48}$ que matériels. En outre, en qualité de juge du suivi des mesures prononcées par la juridiction de jugement, le JAP dispose à l'instar du juge d'instruction, de larges pouvoirs coercitifs comme la possibilité de délivrer des mandats ou d'être assisté par les autorités policières. En revanche et contrairement, au juge d'instruction saisi in rem, la juridiction de l'application des peines peut se saisir d'office. Cette prérogative exorbitante du droit commun, déroge a fortiori à la détermination de l'objet du litige, qui appartient dans le procès pénal par nature au ministère public. Or, le soupçon de partialité ne pèse-t-il pas sur le juge, qui est à la fois juge de l'initiative et juge du litige ${ }^{49}$ ? La prérogative d'auto-saisine est ainsi prévue par les dispositions

\footnotetext{
${ }^{47}$ V. en ce sens : L. CADIET, J. NORMAND, S. AMRANI-MEKKI, "Théorie générale du procès », Thémis, PUF

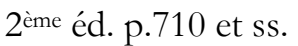

${ }^{48}$ Les juridictions de l'application des peines peuvent faire procéder ou procéder à toutes mesures utiles sur l'ensemble du territoire.

49 B.ROLLAND, «Réflexions sur la saisine d'office», in Mélanges en l'honneur du Professeur Wiederker, Dalloz 2009, p. 685.
} 
générales de l'article 712-4, c'est pourtant sur les fondements spéciaux du Sme ${ }^{50}$ puis de la semiliberté que la Cour de cassation a dit n’y avoir lieu à renvoi devant le Conseil Constitutionnel. La cour de cassation a en effet estimé que " la décision de procéder à ce débat ne préjuge pas de la révocation de la semi-liberté et n'affecte donc pas l'impartialité du juge $»^{51}$.Contrairement au juge des enfants dont la situation a été censurée par le Conseil Constitutionnel en raison de la violation de la séparation de l'autorité de poursuite et de jugement ${ }^{52}$, l'auto saisine du JAP en matière de révocation pose moins de difficultés en ce que le juge n'est amené qu’à apprécier la caractérisation des incidents et la proportionnalité de la sanction, il serait néanmoins préférable que le JAP soit saisi par le ministère public auquel serait soumis l'appréciation des difficultés d'exécution. Une fois l'objet du litige délimité à l'initiative du juge, du ministère public ou de la partie condamnée, le processus de détermination de la décision ne diffère en rien des juridictions pénales. L'appel de la décision rendue par la juridiction du premier degré pourra donc être interjeté soit par la personne condamnée, soit par le procureur de la République ou le parquet général, à l'exception de la partie civile, laquelle n'est pas partie au procès devant les juridictions de l'application des peines.

21-. Les dispositions spécifiques liées à l'appel sont envisagées sous les articles 712-11 à 712-15 et précisent notamment les règles applicables selon que la procédure est soumise au débat contradictoire ou bien écrite ainsi que les délais applicables qui varient selon la qualité de l'appelant. L'étendue de l'effet dévolutif de l'appel devant les juridictions pénales est précisée par les dispositions de l'article 515 du code de procédure pénale. La matière pénale présente en outre la spécificité de se voir appliquer la prohibition de la reformatio in pejus, selon laquelle, il n'est pas possible d'aggraver le sort de celui qui interjette appel. On a pu douter de leur transposition au droit de l'application des peines en raison du silence gardé par le Code de procédure pénale au stade postsentenciel et du champ d'application limité de l'article 515 du code de procédure pénale au stade du seul jugement. L'effet dévolutif, lorsqu'il est évoqué en post-sentenciel, ne l'est que par des textes de source décrétale, aux articles D. 49-42, D. 49-43 et D. 49-44-1 du Code de procédure pénale. C'est pourquoi, ne s'estimant pas liée par les principes processuels applicables au procès pénal, la chambre criminelle avait dans un premier temps accordé les pouvoirs les plus les plus larges aux juridictions d'appel, qui en vertu de leur pouvoir d'appréciation étaient autorisées à revoir à la hausse ou à la

\footnotetext{
${ }^{50}$ Cass. crim 8 juin 2011, $\mathrm{n}^{\circ} 11-90045$, non-lieu à renvoi QPC.

${ }^{51}$ Cass. crim. $1^{\text {er }}$ sept. 2011, nº 11-90070, AJ Pénal 2013, p. 484, obs. M. Herzog-Evans.

${ }^{52}$ Cons. Const. 8 juill. 2011, n 2011-147, QPC
} 
baisse les réductions de peine supplémentaires accordées par le JAP ${ }^{53}$. Par arrêt 12 février $2014^{54}$ la chambre criminelle a opéré un revirement de jurisprudence concernant les pouvoirs du Président de la Chap. La chambre criminelle relevant d'office le moyen de cassation affirme que saisi du seul appel du condamné interjeté contre une ordonnance portant octroi de réductions supplémentaires de peine, le Pt de la CHAP ne peut aggraver le sort de l'appelant. Bien que l'arrêt n'ait pas été rendu au visa de l'article 515 du code de procédure, mais sur le fondement de l'article 721-1 du Code de procédure pénale et des grands principes que sont l'effet dévolutif de l'appel et la prohibition de l'aggravation du sort de l'appelant sur son seul appel. L'on peut déduire de cet arrêt, l'application d'un principe général au droit de l'application des peines. Depuis, non seulement la chambre criminelle a confirmé la prohibition de la réformatio in pejus devant les juridictions de l'application des peines $^{55}$, mais elle a même rendu des décisions au visa de l'article 515 du Code de procédure pénale venant corriger et encadrer les textes spécifiques applicables au droit de l'application des peines ${ }^{56}$ par emprunt direct à l'effet dévolutif applicable aux juridictions pénales. Ces décisions illustrent l'ascendance croissante du droit commun en droit de l'application des peines qui se heurte parfois à la difficulté liée à la localisation des débats.

\section{B/ La localisation}

22-Certaines décisions du $\mathrm{JAP}^{57}$ sont rendues «en CAP», laquelle a pour principale fonction de donner son avis. Par sa localisation- au sein de l'établissement pénitentiaire- et sa composition -en sont membres de droit le JAP qui la préside, le procureur de la République et le chef d'établissementla CAP n'est pas une juridiction « ordinaire». Certains auteurs n'ont pas hésité à la qualifier de «quasi-juridiction $»^{58}$. Si la CAP a bien une apparence juridictionnelle, la délimitation entre la fonction juridictionnelle exercée par le JAP et les fonctions exercées par l'administration pénitentiaire en tant que membre de droit ou membres obligatoires est source de confusion. La CAP est ainsi destinataire de tout changement concernant le régime de détention de la personne détenue. Le juge

${ }^{53}$ Cass. crim. 7 mars 2007, Bull. crim. n 75 ; AJpénal 2007 p. 289 ; Dr Pénal 2007, chron. 2, n 38, obs. E. Garçon.

${ }^{54}$ Cass. crim. 12 février 2014, n 13-81.683 ; Dr pénal 2014 comm, 83, note E. Bonis Garçon.

${ }^{55}$ Cass. crim. 10 déc. 2014, $\mathrm{n}^{\circ}$ 14-81.056, Dr pénal 2015, chronique un an du droit de la peine, $\mathrm{n}^{\circ} 24$, E. Bonsi-Garçon.

${ }^{56}$ Cass. crim. 18 déc. 2013, AJ pénal 2014 p. 196, obs. M. Herzog-Evans ; Cass. crim. 10 déc. 2014, n $14-$ 81.056, Dr pénal mars 2015, p. 19, chronique : un an du droit de la peine, , obs. E. Bonis-Garçon.

${ }^{57} \mathrm{La}$ CAP est ainsi consultée par le JAP sur les décisions relatives aux réductions de peines (retrait des CRP, réductions de peines supplémentaires), autorisation de sortie sous escorte, permissions de sortir.

${ }^{58}$ M. HERZOG-EVANS, Droit de l'exécution des peines, Dalloz action, 2012, spéc. Chap. 141. 
statuera le plus souvent au vu d'un dossier élaboré unilatéralement par l'administration pénitentiaire. Son rôle ne s'analyse donc pas seulement comme un organe consultatif mais aussi comme un organe d'instruction, qui à défaut de prendre des décisions contraignantes, participe activement à leur élaboration ${ }^{59}$.. En outre, la procédure suivie devant la CAP est dérogatoire au droit commun. Non seulement la personne condamnée ne comparaît pas devant la CAP, mais encore elle ne bénéficie ni de la représentation, ni de l'assistance d'un avocat. Enfin, la composition même de la CAP tend à fragiliser le JAP dans les signes distinctifs du juge que sont l'impartialité et l'indépendance. La juridictionnalisation de l'exécution des peines n'a donc pas produit la plénitude de ses effets devant la CAP. Deux raisons peuvent expliquer la situation particulière des décisions prises en CAP. La première est historique et consiste dans les traces laissées par les étapes successives de la juridictionnalisation. L'on observera en effet que les mesures ${ }^{60}$ relevant de la CAP ont été celles qui, historiquement ont été épargnées dans un premier temps par la juridictionnalisation, pour réintégrer dans un second temps seulement, le champ des décisions de justice. Comme une piqûre de rappel, ce sont ces mesures qui sont prises par la voie d’ordonnance selon une procédure «allégée ». La seconde a trait aux incertitudes sur l'applicabilité de l'article 6 de la Conv. EDH aux procédures postsentencielles.

23. En conséquence, la CAP se laisse difficilement appréhender par les critères organiques et matériels traditionnels permettant de définir une juridiction. Cependant face notamment à la juridictionnalisation croissante des autorités, l'on peut avec d'autres auteurs s'interroger sur la pertinence de l'approche classique de la juridiction qu'il est préférable de délaisser au profit d'une approche fonctionnelle ${ }^{61}$. La CAP est avant tout un lieu d'échanges, permettant au JAP d'apprécier librement s'il doit suivre ou non les avis qui auront été émis en son sein et de rendre par voie d'ordonnance une décision susceptible d'appel. La CAP est à mi-chemin entre l'organe doté de simples attributions consultatives et le tribunal au sens conventionnel qui est défini, comme «tout organe à qui il appartient de trancher sur la base de norme de droit et à l'issue d'une procédure organisée, toute question relevant de sa compétence ${ }^{62}$.

\footnotetext{
${ }^{59}$ En ce sens : Y. JOSEPH-RATINEAU, «L'avenir de la commission de l'application des peines à l'aune de la jurisprudence du Conseil d'Etat », à paraître.

${ }^{60} \mathrm{~V}$. note 55.

${ }^{61}$ En ce sens : L. MILANO, «Qu'est-ce qu'une juridiction ? la question a-t-elle encore une utilité ?», RFEDA, 2014, pp. 1119-1130.

${ }^{62}$ CEDH, 22 oct. 1984, SRAMEK C/ AUTRICHE, §36.
} 
24- L'impulsion réformatrice pourrait venir d'une disposition de la loi du 15 août 2014 dans sa partie consacrée à l'exécution des peines ${ }^{63}$, passée relativement inaperçue, qui est selon nous annonciatrice d'un changement de nature de la CAP. A compter du $1^{\text {er }}$ janvier 2015, toutes les personnes détenues exécutant une ou plusieurs peines privatives de liberté d'une durée totale inférieure ou égale à 5 ans et ayant atteint les deux-tiers de leur peine, qui n'ont pas bénéficié d'un aménagement de peine, doivent voir leur situation obligatoirement examinée en CAP, afin que le JAP apprécie s'il y a lieu qu'elles bénéficient d'une sortie encadrée. La mesure étant soumise à l'accord de la personne condamnée, la loi a prévu son audition et que soit rendu possible le recueil des observations de son avocat devant la CAP. Il s'agit de la nouvelle procédure de libération sous contrainte décrite sous l'article 720 du Code de procédure. Ce dernier dispose dans son dernier alinéa que «il (le juge) peut ordonner la comparution de la personne condamnée devant la commission de l'application des peines afin d'entendre ses observations et, le cas échéant, celles de son avocat. ce dernier peut également transmettre des observations écrites au juge de l'application des peines ». Les intentions du législateur quant à l'achèvement de la juridictionnalisation devant la CAP sont loin d'être claires et il ne semble guère, tout comme le législateur du 9 mars 2004, que le législateur du 15 août 2014 ait souhaité retenir devant la CAP une procédure contradictoire. Le législateur ne semble pas avoir anticipé les effets de la pénétration de certaines des garanties du procès équitable au sein de la CAP. Son avenir juridictionnel est en définitive entre les mains des magistrats de l'application des peines ${ }^{64}$ selon les pratiques mises en place. Or parmi les points d'achoppement de la généralisation de la comparution de la personne condamnée assistée de son avocat devant la CHAP est la mise à disposition d'un greffier. L'accès à l'avocat et la présence d'un greffier devant la CAP seraient, parmi les traits différentiels de l'existence d'une juridiction, ceux les plus saillants.

25. Pour conclure, il est grand temps que les procédures devant les juridictions de l'application des peines puissent être encadrées par les grands principes processuels. Les dérogations procédurales décrites ne sont plus justifiées ni par la localisation des débats, ni la crainte d'un contentieux trop volumineux et sont devenues à l'inverse source de désordre par l'adhésion des juridictions internes à des définitions de la sanction pénale discordantes selon qu'elles sont d'inspiration conventionnelle ou

${ }^{63}$ P. PONCELA, « les peines extensibles de la loi du 15 août 2014, RSC 2014, n³ 3, p. 611.

${ }^{64} \mathrm{~V}$. pour les modalités procédurales : Circulaire du 26 décembre 2014 de présentation des dispositions de la loi nº2014-896 du 15 août 2014 relative à l'individualisation des peines et renforçant l'efficacité des sanctions

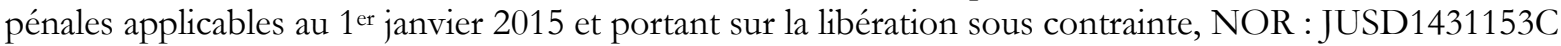


constitutionnelle et dont l'enjeu est l'applicabilité des garanties fondamentales devant les juridictions des peines. A tire d'exemple, il ne semble plus y avoir d'obstacle ni théorique, ni technique à affirmer le droit à la comparution de la personne condamnée devant la Chap. Les décisions commentées montrent que l'intégration des procédures devant les juridiction de l'application des peines au droit commun du procès est en marche.Ce qui pose la question subséquente des limites de la juridictionnalisation et de savoir in fine si la juridictionnalisation doit nécessairement s'accompagner de la transposition de l'ensemble des règles du procès pénal aux juridictions de l'application des peines ou seulement certaines d'entre elles. Pour y répondre, il conviendrait enfin de définir l'objet du procès en application des peines. L'espoir réside dans la réflexion initiée à la demande de Madame le garde des sceaux, dans le sillon de la conférence de consensus, par la Commission Cotte sur la refonte du droit des peines.

Aix-en-Provence le 7 septembre 2015 